\title{
Information Organization Activities in the Knowledge Management Setting
}

Johnson Mulongo Masinde

Information Management, Central China Normal University

China

Otuoma Sanya

Karatina University

Kenya

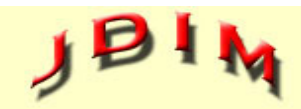

Journal of Digital

Information Management

\begin{abstract}
This study examines information organization activities in the knowledge management environment. The investigation commences with the long-drawn debate on the concept of Knowledge Management and the literature that has hitherto reviewed it. Furthermore, the study delves into how conventional bibliographic information retrieval techniques and expert search systems have heretofore been applied in the organization and retrieval of digital resources and if they can be employed in the Knowledge management setting. It is contended that though the procedures, tools, and techniques are effectual and productive, they cannot be utilized in a knowledge intensive environment. Accordingly, the study puts forth a generic knowledge management framework that can be applied in a knowledge management setting. In addition, it proposes novel research gaps in the context of information organization.
\end{abstract}

Subject Categories and Descriptors: [I.2.4 Knowledge Representation Formalisms and Methods] [H.3 Information Storage and Retrieval]

General Terms: Knowledge Management, Information Processing, Digital Content

Keywords: Information Organization, Knowledge Management Systems, Digital Libraries, Knowledge Management Environment

Received: 18 August 2020, Revised 21 October 2020, Accepted 3 December 2020

Review Metrics: Review Scale: 0-6, Review Score: 5.12, Inter-reviewer consistency: $86 \%$

DOI: $10.6025 / \mathrm{jdim} / 2021 / 19 / 1 / 1-9$

\section{Introduction}

Even though studies regard knowledge management as a management concept in the discipline of business, it has captivated scholars, including those from information science, information technology, and artificial intelligence. As a consequence, innumerable volumes of scholarly content in the area have been published and presented in conferences. Similarly, it has resulted in a myriad of definitions and meanings overtime. Among the many definitions, the most consistently used is the "process of capturing, enhancing, sharing, and capably consuming organizational knowledge (Nazim \& Mukherjee, 2016b; Sousa \& Rocha, 2019). Fundamentally, knowledge management is the management of both the organization's tacit and explicit knowledge resources to realize organizational objectives. Accordingly, Nazim and Mukherjee (2016) analyzed several meanings of knowledge management and listed a number of knowledge management characteristics as itemized below:

I. Knowledge management encompasses knowledge resources from both internal and external sources.

II. Knowledge management methods engrain, and amass knowledge resources in business products, processes and services.

III. The main objective of knowledge management systems is to further growth, share and transfer knowledge resources within the organization

IV. Knowledge management systems evaluate the knowledge resources of the organization and their effects overtime.

Acknowledge management system seeks to present a systematic, objective, and expert view of how computer 
technologies capture, transfer and share knowledge resources in organizations by connecting human expertise and documented knowledge resources in aunified knowledge management system(Johnson Mulongo Masinde \& Jin, 2020).

Some studies have however, pointed out that knowledge management could be another management craze that would evanesce with time. Córdova and Gutiérrez (2018)assert that novel concepts such as Business Processes Reengineering and Total Quality Management etc., have revealed themselves as fads over time. Several studies have also supported the notion that knowledge cannot be managed and that knowledge management systems are only executing the roles of information management systems (Barão, de Vasconcelos, Rocha, \& Pereira, 2017). This study digs deep into the distinctive aspects of knowledge management and information management. It later develops a generic knowledge management model that can be applied in a knowledge management setting. The subsequent section briefly discusses information organization in perspective.

\section{Information Organization Activities in Perspective}

In whichever way we may refer to it, whether a valueadded version of information management or knowledge management, of significance is the information; which feat of a system is dependent. Such a system's probable success will principally depend on how we can expertly capture, organize and disseminate different kinds of information resources as per the user needs(Johnson M. Masinde, Wambiri, \& Chen, 2020).
Information organization builds conceptual frameworks for the organization and retrieval of information, analysis of systems, their purposes, standards, formats, structures, and vocabularies (Willer \& Dunsire, 2013). It includes the description of documents, indexing, and classification and essentially addresses the activities carried out and the tools used in ensuring discovery and access to information resources (Chatterjee, 2017a).

The information organization process's main stages are capturing, organizing, value addition, and dissemination of information resources (Chatterjee, 2017a). In the first stage, relevant information on the organization's activities and needs is captured. This kind of information is usually in varied forms, sources and formats. Once captured, the information is systematized using tools and techniques such as ranking, filtering, retrieving, cataloguing, indexing, etc.An information organization system's effectiveness is mainly dependent on the appropriate organization of information (Chatterjee, 2017b).

\subsection{Conventional approaches to Information Organi- zation Activities}

Libraries hither to employed several tools to organize and avail information resources for discovery and access. The most commonly used thus far are the classification schemes(Universal Decimal Classification, Bibliographic Classification, Dewey Decimal Classification and the Library of Congress Classification) that have mainly been used in the organization of information materials and the Subject Headings lists such as the Library of Congress Subject Headings that have beenapplied in the construction of catalogues (Willer \&

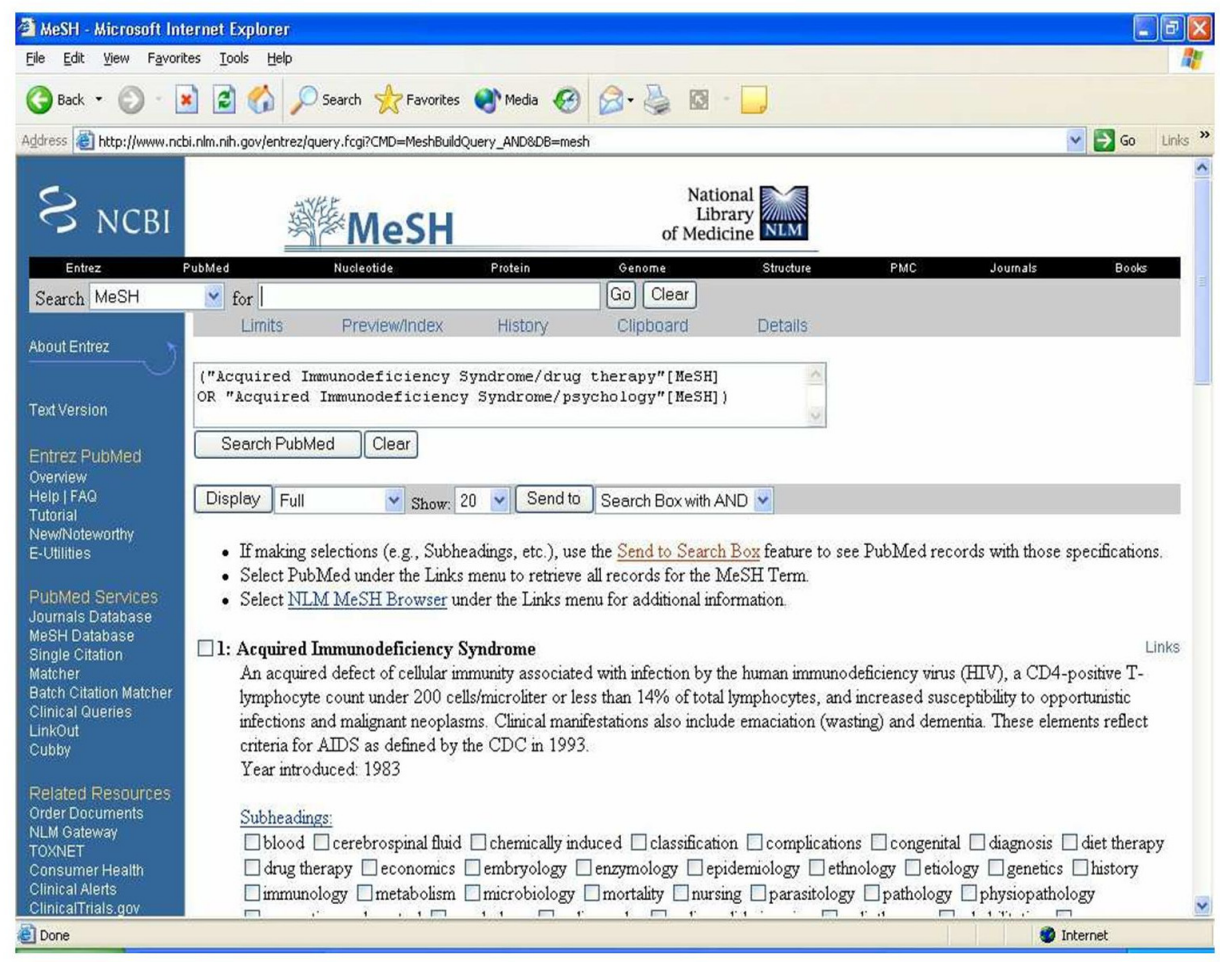

Figure 1. Previously a print screen of the web interface of the National Center for Biotechnology Information (NCBI) which permitted users to select terms from Medical Subject Headings (MeSH) online 
Dunsire, 2013).

Online database systems also used controlled vocabularies like thesauri to construct subject indexes for their information collections. The graphic user interfaces and web interfaces eased the design process of thesauri interfaces for online databases (Chatterjee, 2017b). The screenshot below (Figure 1) shows the web interface of the National Center for Biotechnology Information (NCBI) that formerly enabled users to select terms from the Medical Subject Headings (MeSH) to search for information resources (Kumbhar, 2012).
BUBL (BUlletin Board for Libraries) link was also an Information Organization Service offered by the University of Strathclyde at their Centre for Digital Library Research (CDLR). It provided access to selected web resources through browse and search facilities. In the browse mode, users could access information resources by the Dewey Decimal Class numbers (DDC). Users could also browse by Dewey Index Terms (Kumbhar, 2012).

The print screen below shows a web interface of the BUBL link. It is no longer in use.

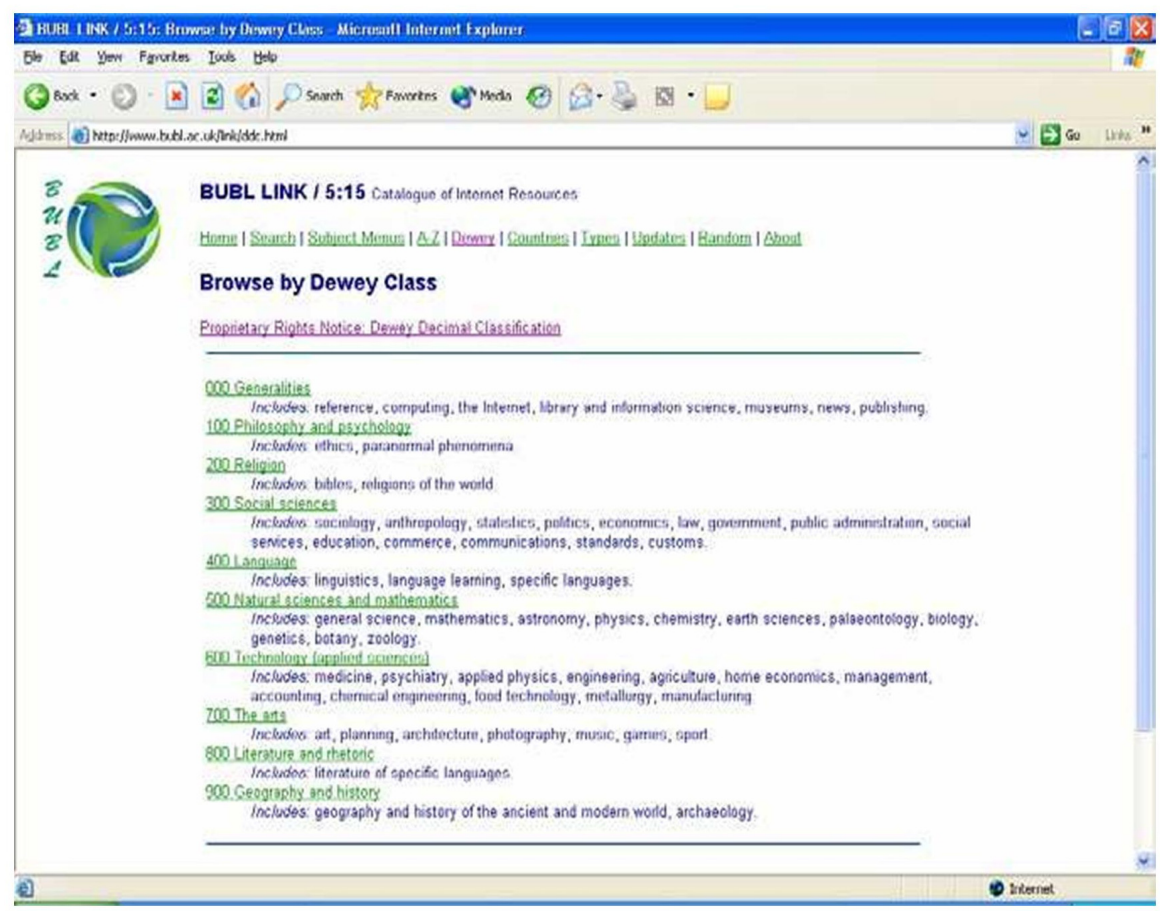

Figure 2. Previously a print screen of BUBL link showing the searching facility by the Dewey Decimal Class numbers (DDC)

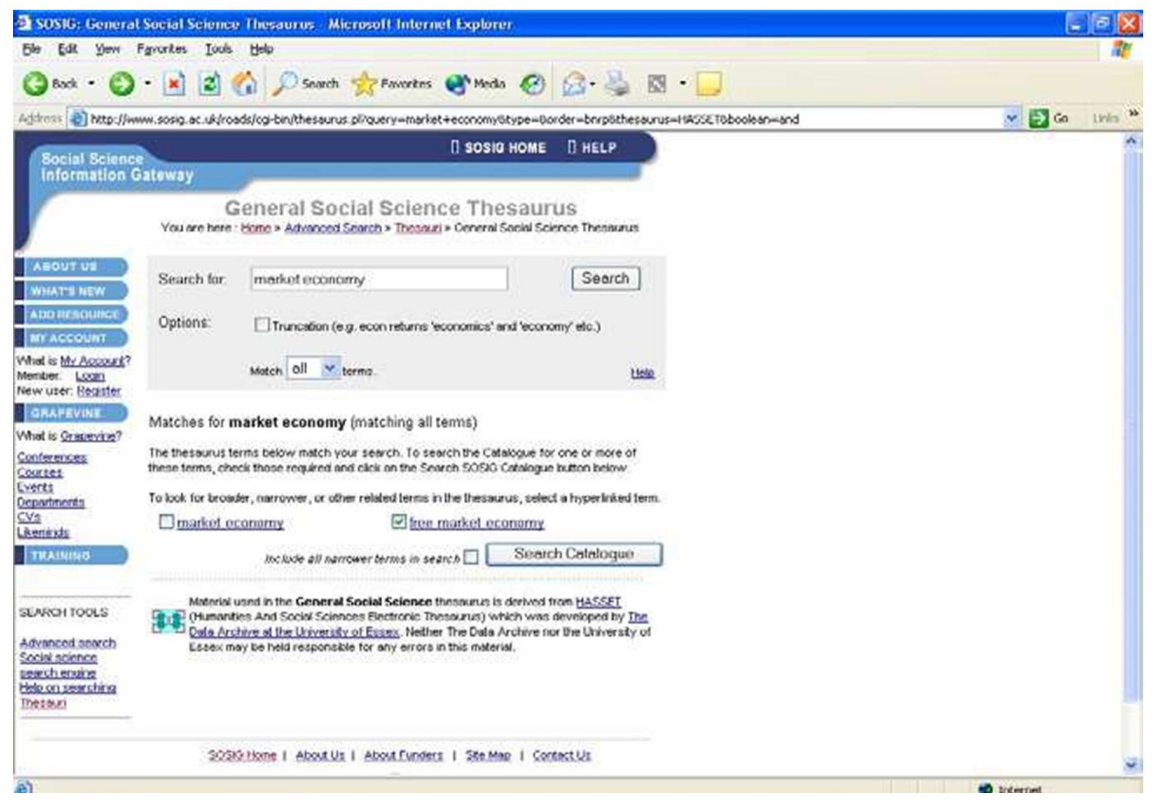

Figure 3. The print screen of the previous Social Science Information Gateway (SOSIG) that permitted users to search using a thesaurus 
The Social Science Information Gateway (SOSIG) was similarly an information organization service with a thesauri interface to permit access to integrated, organized databases, enabling access to high-quality internet resources. It was classified in accordance with the Universal Decimal Classification (UDC) and added into searchable databases. It provided access to all social science information resources (Kumbhar, 2012). Below is the print screen of the previous Social Science Information Gateway (SOSIG).

Even though some of these web interfaces were studies intended to ensure the appropriateness of bibliographic techniques and tools in information organization and retrieval, a number were continued as services.

\subsection{Information Organization Activities with Expert Search Systems}

The web interfaces above were mainly dedicated to bibliographic tools to organize and permit access to information resources. However, today artificial intelligence systems and avant-garde information retrieval expert systems have been employed to design and put forth expert search engines for organization and augmented content retrieval (Papy, 2016). Examples of these specialty search engines include Ask Jeeves (www.askjeeves.com), a meta web browser that uses advanced techniques to organize digital content. Users' results on Ask Jeeves come with other various concepts that are related to the search query and each of the links to specific web content (Chatterjee, 2017b; Papy, 2016).

Kartoo is also another expert search system that uses visualization methods. It ranks among the most powerful in information organization and retrieval (Chatterjee, 2017b; Papy, 2016). A query in Kartoo produces a display of visual results. A cursor pointing on any node shows its links with the other nodes in a visual map. Related concepts are also displayed on the screen. The user can select terms from the related concepts to edit the search query. Below is a print screen of kartoo. The exceptional and germaneness of Kartoo is fashioned by a semi validation process. A team of editors also check the relevance of each indexed website.

Open Knowledge Maps is an expert visual search engine for global research output. Users on the platform can create knowledge maps of research topics on any given topic by conducting a search using a keyword. Knowledge maps provide an immediate overview of a discipline by displaying the major areas at a glance, with relevant research publications and concepts related to each area. To enhance extensive collaboration, the platform is built on open infrastructure using the principles of open science with content, data and platform source code published under Creative Commons Attribution 4.0 International License. Content is harnessed from multiple sources including BASE and PubMed.

Another of the expert search systems is Vivisimo (www.vivisimo.com), which uses clustering methods to retrieve results both on the queried searches and related concepts (Chatterjee, 2017b; Papy, 2016). A screenshot of the interface of Vivisimo is shown below. The results of a search query are visually displayed. A user can browse through the nodes to see relationships and is richly served with the terms and a set of clustered concepts linked to internet resources. The user is, therefore,

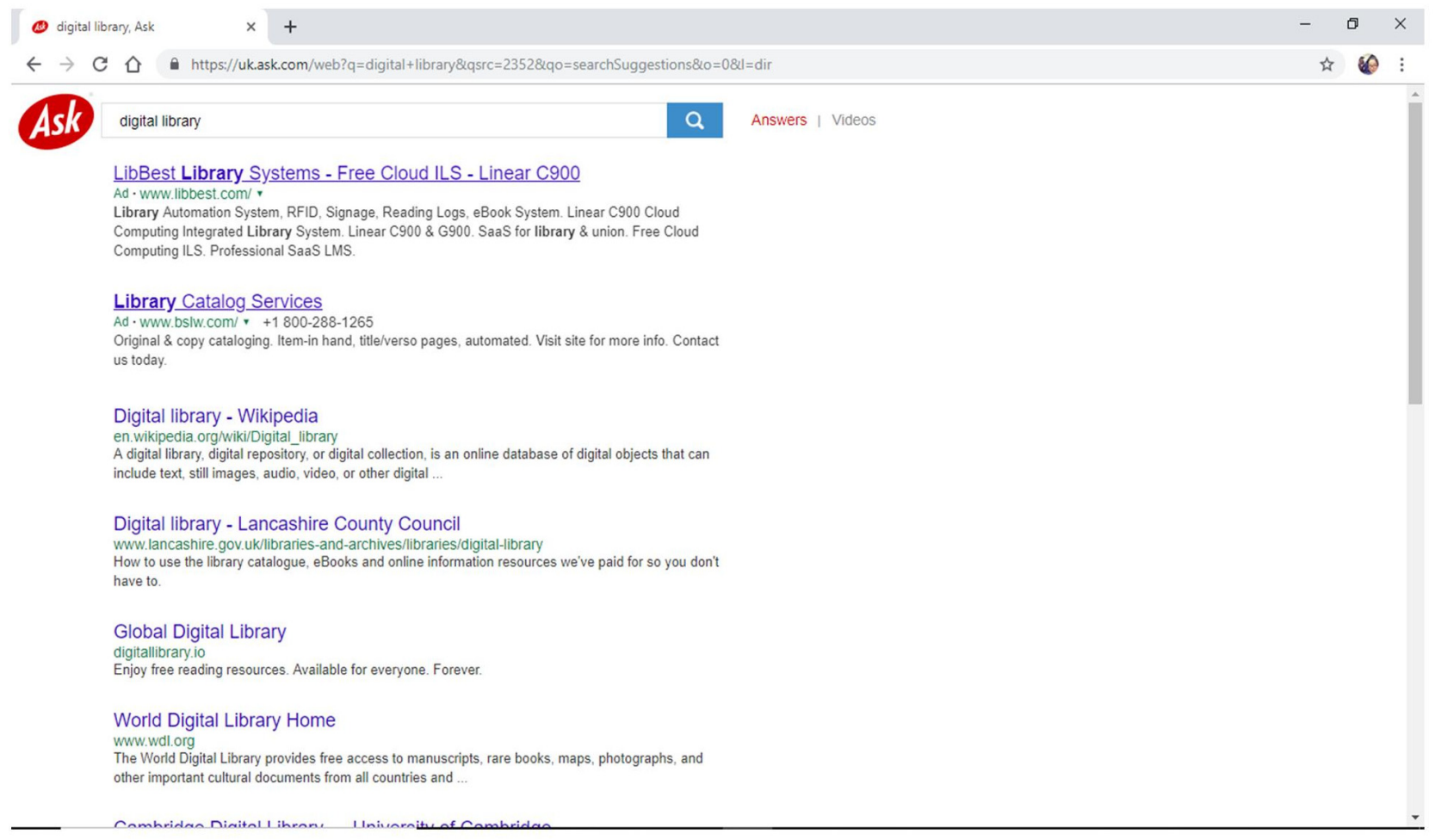

Figure 4. Print screen of Ask Jeeves 


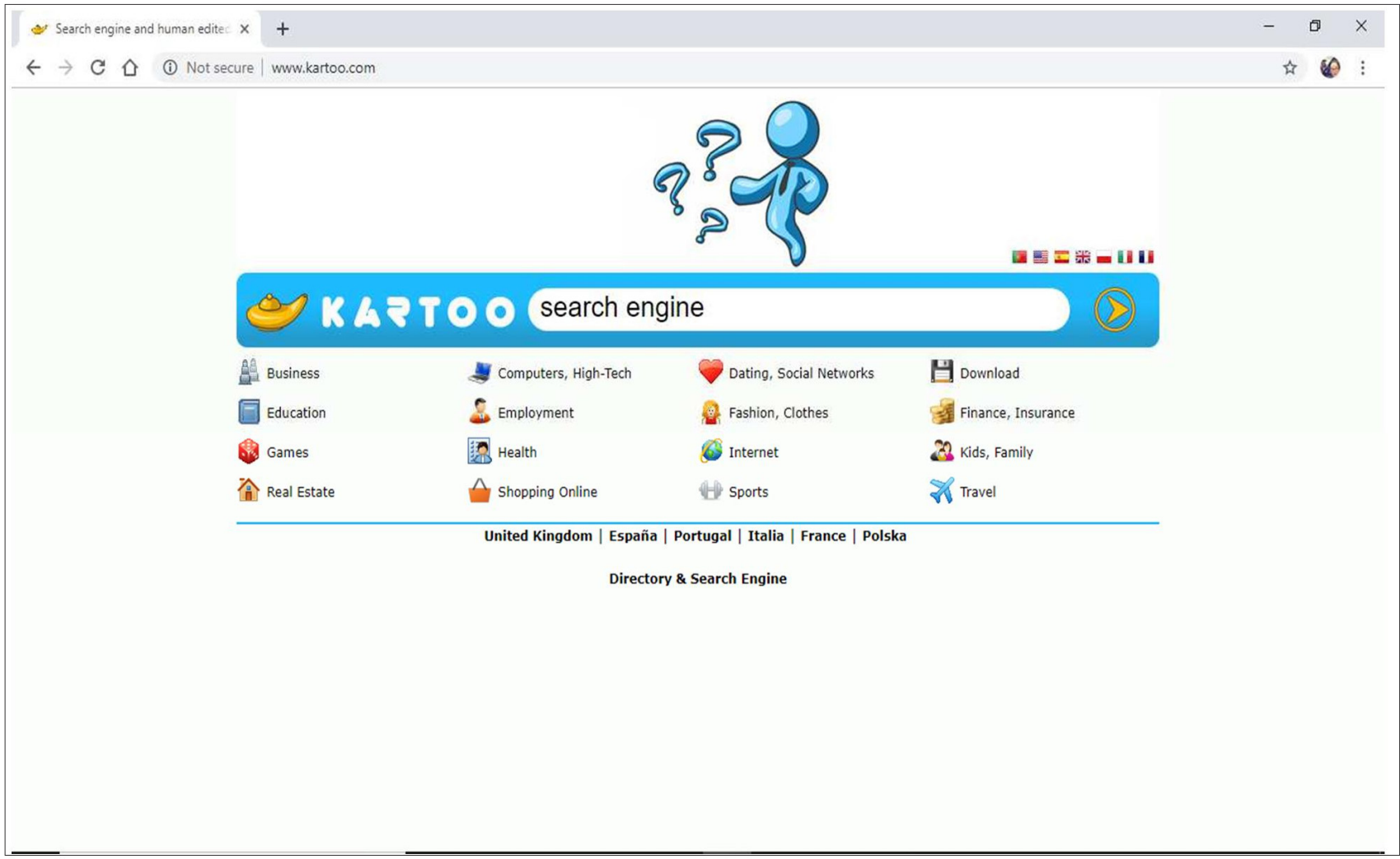

Figure 5. Print screen of kartoo

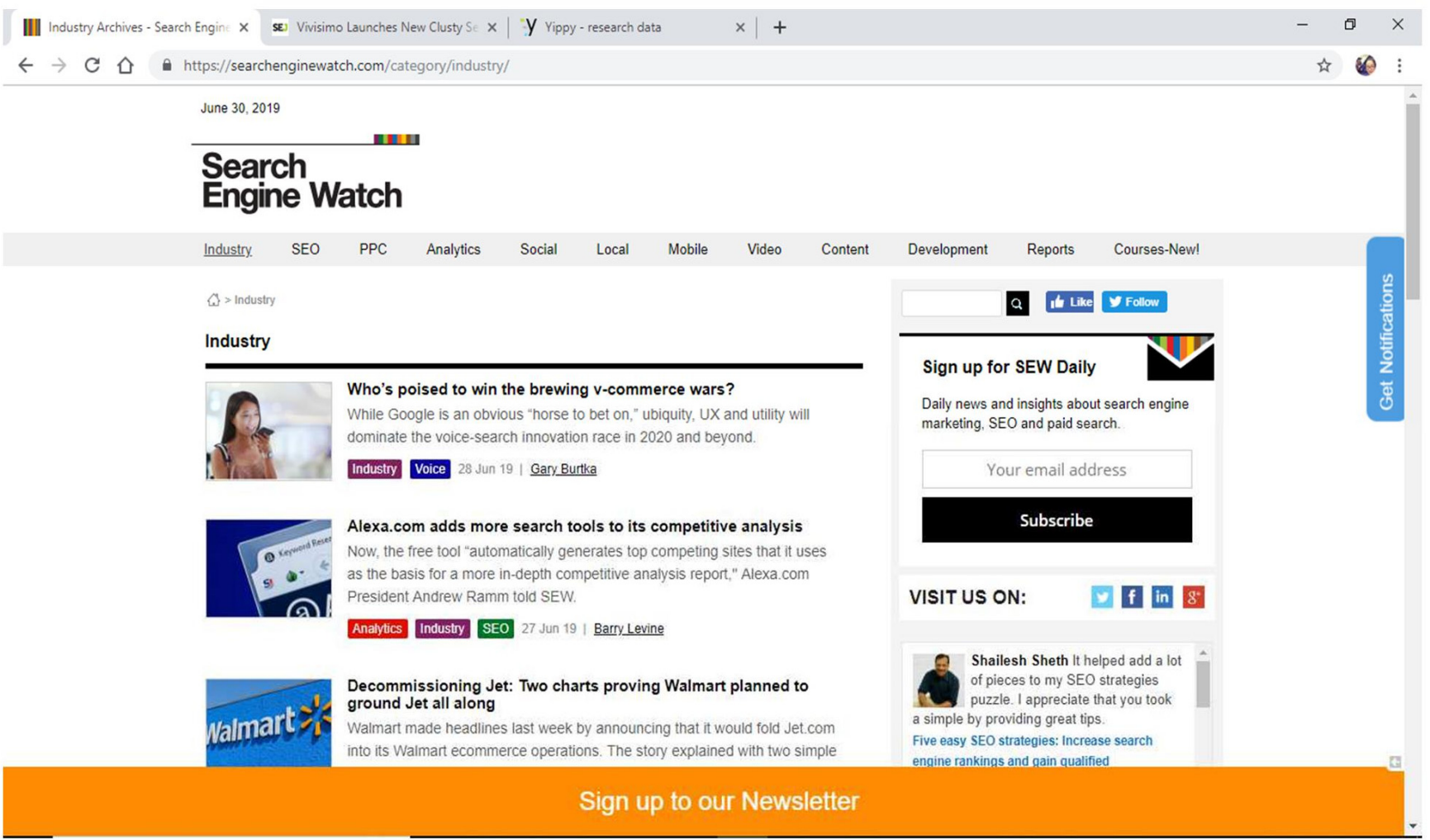

Figure 6. Print screen of vivisimo

able to select the preferred terms and modify the search. The figure below shows a print screen of Vivisimo, which is currently owned by the International Business Machine (IBM).
Yippy is also another expert search engine that groups web results into clusters, initially developed by Vivisimo before being sold to yippy Inc. (Chatterjee, 2017b; Papy, 2016). A print screen of yippy Inc. is shown below. 


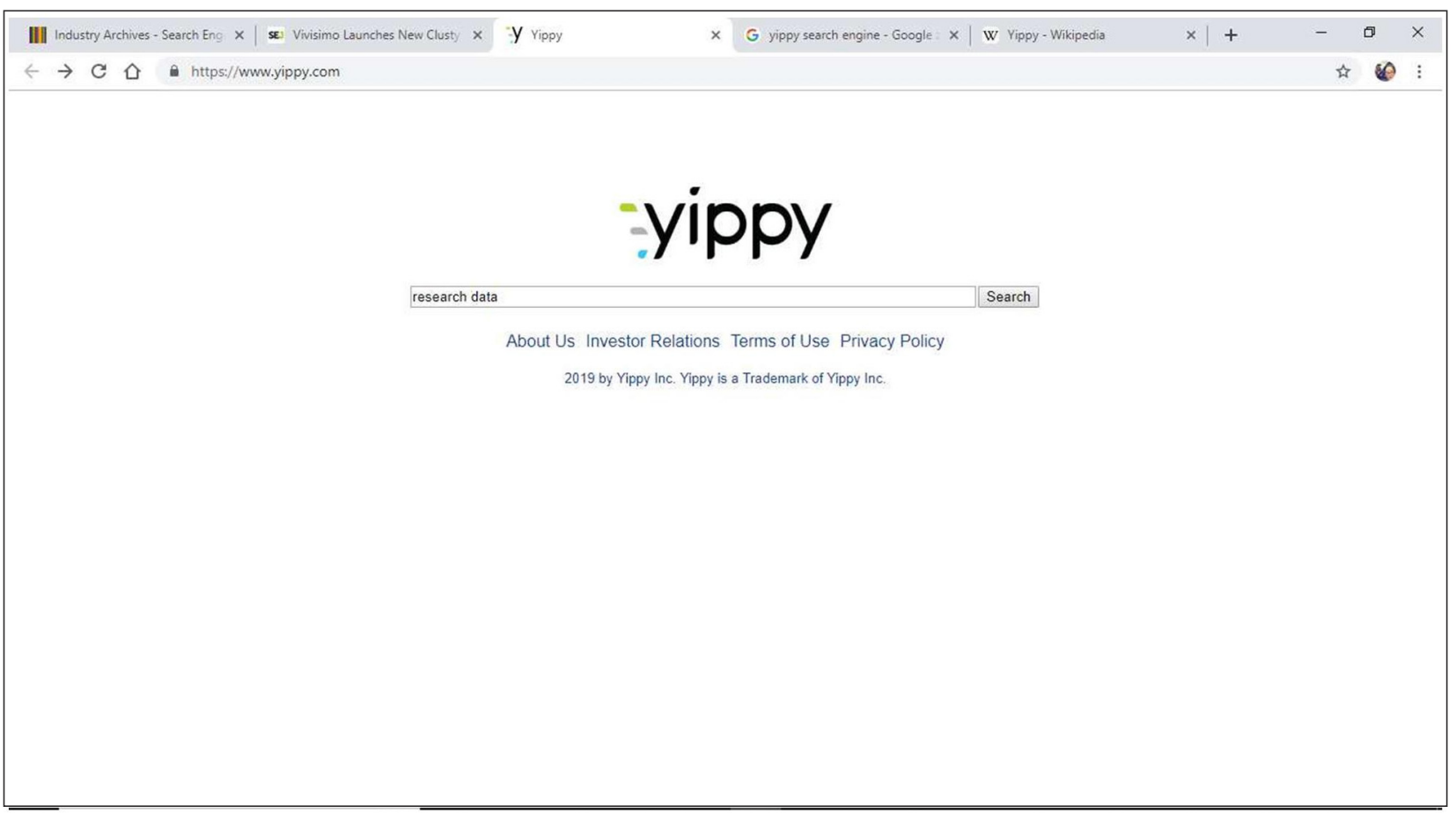

Figure 7. Print screen of yippy Inc

\subsection{Challenges of Expert Search Systems in the Knowledge Management setting}

Even though these systems have proved to be proficient in organization and provision of access to electronic information resources, they have also been thought to have many glitches when used in a knowledge management setting and, therefore, unsuitable (Russell-Rose, Chamberlain, \& Azzopardi, 2018).Some of the glitches to the application of these expert systems in a knowledge management setting are:

(i) Voluminous and explicit amounts of specified information in a knowledge management setting Cooperate environments require massive and detailed amounts of information for operationalization. This kind of information may be available in different forms, both in external and internal databases ranging from minutes of meetings, emails, memos, messages, audio recordings, to information limited to the public domain(Abubakar, Elrehail, Alatailat, \& Elçi, 2019). Not one of the tools briefly discussed above has been applied in the organization of this kind of information. Varied tools and techniques may be required to organize information in hypermedia, textual and alphanumeric form.

\section{(ii) Information Location}

Information in the cooperate organization resides in different localities, places, corporate intranets, web, databases (local and remote databases), etc. (Córdova \& Gutiérrez, 2018). Vast amounts of information may also be available through the library and its integrated management systems. To ensure the organization's proficient operationalization, a framework that would consolidate all these kinds of information must be built. To organize the information, some form of index or metadata has to be constructed, which would permit access to the information from anywhere and in whichever format as per the user rights.

\section{(iii) Organizational culture and work practices}

Information organization tools and techniques discussed in this paper apply to the broader user community (Papy, 2016). In a knowledge management environment, information is explicit to particular roles/activities mainly for the organization's staff with the overall objective of meeting the set goals (Marchiori \& Franco, 2019). This means organizations need institutionalized and personalized information. Therefore, the critical considerations while constructing an information organization system for the organization are the overall organizational culture, practices, and framework in terms of the purposes or the tasks at hand and the people going to use the information organization system (Córdova \& Gutiérrez, 2018).

The subsequent section puts into perspective the knowledge management setting and further proposes a generic model for the knowledge management setting.

\section{Knowledge Management in Perspective}

For some time now, several studies have been of the inkling that management of knowledge is not conceivable for the reason that it is what we know and is therefore vested in the human mind (Kumbhar, 2012; Nazim \& Mukherjee, 2016b). Hwang, Lin and Shin (2018) distinguishes information and knowledge by asserting that knowledge is the know-how and therefore rests in the mind and when it leaves the mind, it vicissitudes to information. For instance, the messages created for communication purposes constitute information resources of 
different kinds, both formal (Journal articles, books) and Informal (Emails, minutes of a meeting).

After critically analyzing a number of studies, views by professionals in both information management and knowledge management and the terminological dissimilarities between knowledge management and information management, Nazim and Mukherjee (2016) concluded that two pillars support knowledge management. Constructive management of occupational practices and Information management. The authors aver that the first pillar is grounded on the mutual belief of organizational culture under which all the workforce shares the advantages of organizational information. Employees exclusively nurture their know-how, and communities in the organization define how the know-how/expertise will be utilized. The sole purpose of a Knowledge management system is to realize organizational goals(Nazim \& Mukherjee, 2016a).

Therefore, the subsequent section will delve deeper into the knowledge management environment and further propose a generic model for the Knowledge Management environment.

\section{The Knowledge Management System for the Knowl- edge Management setting}

Electronic libraries that can develop content, local and remotely, on distributed servers and have the content discoverable and accessible to every user in the organization can play a significant role in the knowledge management setting (Yang \& Li, 2016). To permit proficient operationalization, organizations capture all the information that is deemed relevant in keeping with their set objectives. The information could come from databases, local intranet, internet, local and remote libraries. This includes information resources on both work culture and local expertise(Wambiri \& Masinde, 2019).

Such a knowledge management system permits user search of knowledge resources or enables access to knowledge resources recommended automatically by the system in accordance with the user needs or tasks of the organization (Córdova \& Gutiérrez, 2018). The knowledge management system then systematizes, refines, and selectively personalizes the knowledge resources in line with the users' or professionals' needs (Johnson Mulongo Masinde \& Jin, 2020).

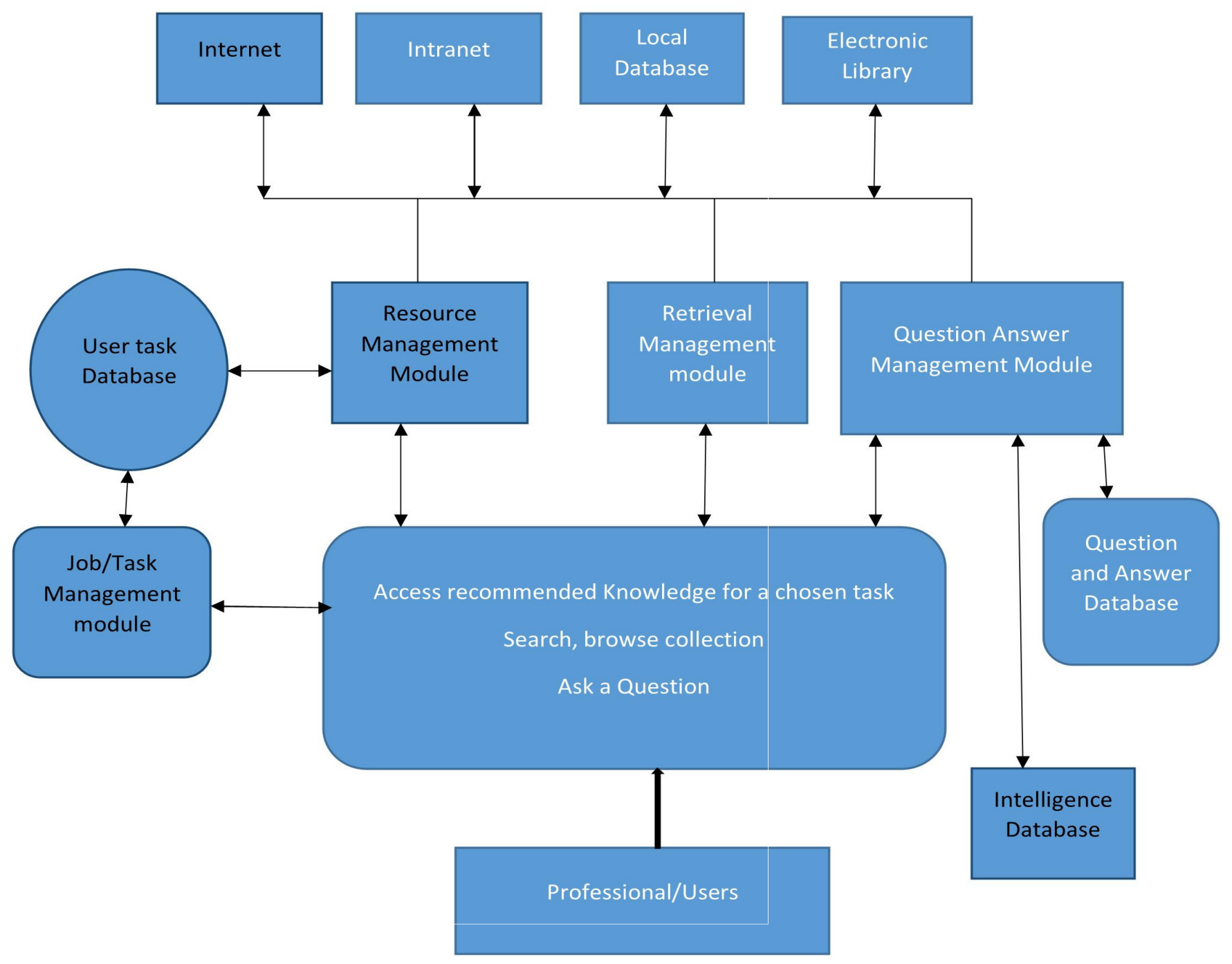

Figure 8 . The knowledge management system for the knowledge management setting 
It equally integrates the knowledge needs of the tasks to be executed in accordance with each user and in the manner in which the tasks are to be executed. Furthermore, it is consistent with the access rights of each professional/user's knowledge and their varied knowledge requirements. Of importance, all the activities are executed behind the scenes, and the user/professional gets access to the requisite knowledge resources upon selecting the task to be performed (Córdova \& Gutiérrez, 2018). The user can also browse through the whole collection and submit queries if they want to know more about specific tasks assigned. The diagram below shows a knowledge management system for a knowledge setting.

\section{Conclusion}

The knowledge management system proposed here is generic and can be made even more proficient by adding knowledge organization and access facilities at a number of levels or sections. For instance, knowledge resources deemed more vital for a certain task may perhaps be displayed on the user screen in a visual or graphic map enabling the user to get an inkling of the general knowledge map of the organization with regard to the needed knowledge resources. Through this, users are able to get an overview of all the knowledge resources and the overall work culture and best practices of the organization. Users are also able to polish their skills since there are a lot of knowledge resources available in the organization.

Designing a suitable ontology for the knowledge management system can improve the task-based recommender and the knowledge search interface. A standard information organization and access tool may not be suitable for the organization though it may form a foundation for development of an ontology. Ontologies are explicated as terms used to describe and represent an area of knowledge resources (Guarascio, Manco, \& Ritacco, 2019). They capture associations and boast management of knowledge resources. A specially designed ontology may not only be used in the organization of knowledge resources but may also form a display of visual map of concepts which may enable the user to frame and revise the queries.

Future studies could investigate the need to audit the knowledge use and user behavior patterns of knowledge resources which may arise from time to time; indirectly by analyzing transaction logs or directly through user surveys. Future studies could therefore use this kind of information to modify and review as deemed necessary the techniques applied in the management of knowledge resources and to design a specific and refined knowledge management system architecture.

\section{References}

[1] Abubakar, A. M., Elrehail, H., Alatailat, M. A., Elçi, A.
(2019). Knowledge management, decision-making style and organizational performance. Journal of Innovation \& Knowledge, 4 (2) 104-114. doi:https://doi.org/10.1016/ j.jik.2017.07.003

[2] Barão, A., de Vasconcelos, J. B., Rocha, Á., Pereira, R. (2017). A knowledge management approach to capture organizational learning networks. International Journal of Information Management, 37 (6) 735-740. doi:https://doi.org/10.1016/j.ijinfomgt.2017.07.013

[3] Chatterjee, A. (2017a). Chapter E - Organization of Information. In: A. Chatterjee (Ed.), Elements of Information Organization and Dissemination (p. 95-98): Chandos Publishing.

[4] Chatterjee, A. (2017b). Chapter Y - Automated Information Organization. In: A. Chatterjee (Ed.), Elements of Information Organization and Dissemination (p. 497526): Chandos Publishing.

[5] Córdova, F. M., Gutiérrez, F. A. (2018). Knowledge Management System in Service Companies. Procedia Computer Science, 139, 392-400. doi:https://doi.org/ 10.1016/j.procs.2018.10.275

[6] Guarascio, M., Manco, G., Ritacco, E. (2019). Knowledge Discovery in Databases. In: S. Ranganathan, M. Gribskov, K. Nakai, \& C. Schönbach (Eds.), Encyclopedia of Bioinformatics and Computational Biology (p. 336341). Oxford: Academic Press.

[7] Kumbhar, R. (2012). 1 - Knowledge organisation and knowledge organisation systems. In: R. Kumbhar (Ed.), Library Classification Trends in the 21st Century (p. 16): Chandos Publishing.

[8] Marchiori, D., Franco, M. (2019). Knowledge transfer in the context of inter-organizational networks: Foundations and intellectual structures. Journal of Innovation \& Knowledge. doi:https://doi.org/10.1016/j.jik.2019.02.001

[9] Masinde, J. M., Jin, C. (2020). A Knowledge Resource Management System (KRMS) for the Academic Community. International Journal of Web Applications, 12 (3) 79-84. doi:0.6025/ijwa/2020/12/3/79-84

[10] Masinde, J. M., Wambiri, D. M., Chen, J. (2020). Gender and cognitive factors influencing information seeking of graduate students at Kenyatta University Library. 2020, 22 (1). doi:10.4102/sajim.v22i1.1154

[11] Nazim, M., Mukherjee, B. (2016a, 2016/01/01/). Chapter 2 - History and Evolution of Knowledge Management Systems. Knowledge Management in Libraries. Chandos Publishing.

[12] Nazim, M., Mukherjee, B. (2016b). Chapter 6 - Knowledge Management Tools for Libraries. In M. Nazim \& B. Mukherjee (Eds.), Knowledge Management in Libraries (p. 115-148): Chandos Publishing.

[13] Papy, F. (2016). 1 - Information Retrieval, Web and Interoperability. In: F. Papy (Ed.), Digital Libraries (p. 128): Elsevier. 
[14] Russell-Rose, T., Chamberlain, J., Azzopardi, L. (2018). Information retrieval in the workplace: A comparison of professional search practices. Information Processing \& Management, 54 (6) 1042-1057. doi:https:/ /doi.org/10.1016/j.ipm.2018.07.003

[15] Sousa, M. J., Rocha, Á. (2019). Strategic Knowledge Management in the Digital Age: JBR Special Issue Editorial. Journal of Business Research, 94, 223-226. doi:https://doi.org/10.1016/j.jbusres.2018.10.016

[16] Wambiri, D. M., Masinde, J. M. (2019). Utilization of Mobile Phone and the Ethical Implications on University and Government Services: a Case of Post Graduate Students at Kenyatta University, Kenya. Paper presented at the Proceedings of the 12th International Conference on Theory and Practice of Electronic Governance, Melbourne, VIC, Australia. https://doi.org/10.1145/ 3326365.3326407

[17] Willer, M., Dunsire, G. (2013). 1 - Bibliographic information organization: a view from now into the past. In: M. Willer \& G. Dunsire (Eds.), Bibliographic Information Organization in the Semantic Web (p. 1-83): Chandos Publishing.

[18] Yang, S. Q., Li, L. (2016). Chapter 6 - Evolving Digital Library and Library Digitization. In: S. Q. Yang \& L. Li (Eds.), Emerging Technologies for Librarians (p. 69-102): Chandos Publishing. 